Research Article

\title{
On the Derivation of Nonclassical Symmetries of the Black-Scholes Equation via an Equivalence Transformation
}

\author{
Winter Sinkala $(\mathbb{D}$ \\ Department of Mathematical Sciences and Computing, Faculty of Natural Sciences, Walter Sisulu University Private Bag X1, \\ Mthatha 5117, South Africa \\ Correspondence should be addressed to Winter Sinkala; sinkalaw@gmail.com
}

Received 11 October 2020; Revised 22 November 2020; Accepted 3 December 2020; Published 28 December 2020

Academic Editor: Taha Aziz

Copyright (c) 2020 Winter Sinkala. This is an open access article distributed under the Creative Commons Attribution License, which permits unrestricted use, distribution, and reproduction in any medium, provided the original work is properly cited.

The nonclassical symmetries method is a powerful extension of the classical symmetries method for finding exact solutions of differential equations. Through this method, one is able to arrive at new exact solutions of a given differential equation, i.e., solutions that are not obtainable directly as invariant solutions from classical symmetries of the equation. The challenge with the nonclassical symmetries method, however, is that governing equations for the admitted nonclassical symmetries are typically coupled and nonlinear and therefore difficult to solve. In instances where a given equation is related to a simpler one via an equivalent transformation, we propose that nonclassical symmetries of the given equation may be obtained by transforming nonclassical symmetries of the simpler equation using the equivalence transformation. This is what we illustrate in this paper. We construct four nontrivial nonclassical symmetries of the Black-Scholes equation by transforming nonclassical symmetries of the heat equation. For completeness, we also construct invariant solutions of the Black-Scholes equation associated with the determined nonclassical symmetries.

\section{Introduction}

There is a lot of interest in the search for nonclassical symmetries of differential equations. This is because nonclassical symmetries lead to new solutions of differential equations, solutions not obtainable directly from classical Lie point symmetries of the equation. Nonclassical symmetries are however not easy to find due to the complex nature of the governing equations that define the associated infinitesimals.

In the search for nonclassical symmetries, we typically make assumptions on the nature of the associated infinitesimals to simplify the governing equations. This approach is complemented by the use of Heir-equations [1-5]. These are nonlinear equations that result from iterations of the nonclassical symmetries method and inherit the Lie algebra of symmetry generators of the given equation. Similarity solutions of Heir-equations yield nonclassical symmetries of the original equation. Other approaches for finding nonclassical symmetries of differential equations have been proposed [6-8].
The primary aim of this paper is to propose the use of equivalent transformations to augment methods for finding nonclassical symmetries of differential equations. We illustrate that when a given differential equation is equivalent to another (target) differential equation through an invertible point transformation, one can construct nonclassical symmetries of the given equation from those of the target equation through the point transformation. We use this routine to construct nontrivial nonclassical symmetries of the famous Black-Scholes equation [9]:

$$
u_{t}+\frac{1}{2} \sigma^{2} x^{2} u_{x x}+r x u_{x}-r u=0
$$

The nonclassical symmetries method was devised by Bluman and Cole in 1969 [10] to find new exact solutions of the heat equation. The method has since been applied to many different types of problems involving partial differential equations (see, for example, [11-13]). Under the nonclassical symmetries method, we seek invariance of a given partial differential equation (PDE) considered together with the invariant surface condition. The method is an 
extension (among other extensions [14-16]) of the classical symmetries method [17-23] for finding exact and special solutions of PDEs.

We shall, in this paper, restrict our discussion to secondorder $(1+1)$ PDEs of the form

$$
\Delta\left(x, t, u, u_{x}, u_{t}, u_{x x}, u_{x t}, u_{t t}\right)=0,
$$

where $(x, t) \in \mathbb{R}^{2}$ are independent variables and $u \in \mathbb{R}$ is the dependent variable.

To apply the classical symmetries method to (2), we consider a one-parameter group action on the $(x, t, u)$ space given by its infinitesimals

$$
\begin{aligned}
& \tilde{x}=x+\varepsilon \xi(x, t, u)+O\left(\varepsilon^{2}\right), \\
& \tilde{t}=t+\varepsilon \tau(x, t, u)+O\left(\varepsilon^{2}\right), \\
& \tilde{u}=u+\varepsilon \eta(x, t, u)+O\left(\varepsilon^{2}\right),
\end{aligned}
$$

depending on a continuous parameter $\varepsilon$. This transformation is characterised by its infinitesimal generator:

$$
X=\xi(x, t, u) \partial_{x}+\tau(x, t, u) \partial_{t}+\eta(x, t, u) \partial_{u} .
$$

Equation (2) is invariant under (3) if and only if

$$
\left.X^{(2)} \Delta\right|_{\Delta=0}=0 \text {, }
$$

where $X^{(2)}$ is the second extension of the infinitesimal generator (4). The invariance condition (5) yields governing equations for the infinitesimals $\xi, \tau$, and $\eta$. These equations are necessarily an overdetermined system of linear PDEs, the general solution of which provides the infinitesimals.

The nonclassical symmetries method differs from the classical symmetries method in that we now seek invariance of equation (2) augmented with the invariant surface condition:

$$
\psi=\tau(x, t, u) u_{t}+\xi(x, t, u) u_{x}-\eta(x, t, u)=0,
$$

i.e.,

$$
\begin{aligned}
& \left.X^{(2)} \Delta\right|_{\Delta=0, \psi=0}=0, \\
& \left.X^{(1)} \psi\right|_{\Delta=0, \psi=0}=0 .
\end{aligned}
$$

It can easily be shown that equation (6) is trivially invariant under (3), i.e., the second equation in (7) vanishes identically without imposing any conditions upon $\tau$, $\xi$, and $\eta$. Therefore, the pair of conditions in (7) reduces to the singular condition:

$$
\left.X^{(2)} \Delta\right|_{\Delta=0, \psi=0}=0,
$$

which is the invariance condition for nonclassical symmetries of equation (2). Equation (8) leads to a set of nonlinear, coupled governing equations for the infinitesimals $\xi$, $\tau$, and $\eta$. In instances where these equations can be solved, we obtain nonclassical symmetries of equation (2). The invariance surface condition (6) then leads to a solution ansatz which, when substituted into equation (2), leads to a reduction of the equation, and ultimately invariant solutions of the equation.
The rest of the paper is organised as follows. In Section 2, we present an invertible point transformation that is subsequently used to map the Black-Scholes equation to the heat equation. We also discuss some routines for deriving such transformations. This is followed by derivation of nonclassical symmetries of the heat equation, which we perform in Section 3. In Section 4, we perform transformations of the determined nonclassical symmetries of the heat equation into corresponding nonclassical symmetries of the Black-Scholes equation. The construction of new solutions of the Black-Scholes equation from the determined nonclassical symmetries is carried out in Section 5. Finally, we give concluding remarks in Section 6.

\section{Equivalence Transformation between the Black-Scholes and Heat Equations}

It is well known (see $[17,18,24-27]$ and the references therein) that if a mapping from a given differential equation to another (target) differential equation is an invertible point transformation, then the mapping establishes a one-to-one correspondence between symmetries of the given and target equations. Such mappings are realisable as equivalence transformations between equations that have similar symmetry Lie algebras. An equivalence transformation is essentially a change of variables that maps a differential equation in a given class to another differential equation in the same class.

According to Lie's result of group classification of linear second-order PDEs with two independent variables, the Black-Scholes equation can be reduced to the heat equation,

$$
w_{y}=w_{z z}
$$

by a point transformation, Lie's equivalence transformation,

$$
\begin{aligned}
& z=\alpha(x, t), \\
& y=\beta(t), \\
& w=\varphi(x, t) u,
\end{aligned}
$$

for some functions $\alpha, \beta$, and $\varphi$, with $\alpha_{x} \neq 0$ and $\beta_{t} \neq 0$ (see [24]). Therefore, an equivalence transformation that relates the Black-Scholes equation and the heat equation may be determined by using (10) to write the heat equation in terms of the Black-Scholes equation variables and then comparing the resulting equation with the Black-Scholes equation (see [24]). The functions $\alpha, \beta$, and $\varphi$ follow from the resulting set of determining equations, and we obtain (as in [9])

$$
\begin{gathered}
z=\delta_{1}+\frac{2 \kappa(\ln x-\kappa t)}{\sigma^{2}}, \\
y=\frac{\delta_{2}-2 \kappa^{2} t}{\sigma^{2}}, \\
w(z, y)=\delta_{3} u(x, t) e^{-r t},
\end{gathered}
$$


where

$$
\kappa=r-\frac{1}{2} \sigma^{2}
$$

and $\delta_{i}$ are arbitrary constants.

An equivalence transformation between two equations may also be derived by using Lie point (or contact) transformations admitted by the equations. These provide a means by which to identify equivalent differential equations and to construct equivalent transformations between them when such transformations exist $[17-19,25,26,28,29]$. There are other practical criteria that involve characterising equivalence transformations between partial differential equations in terms of coefficients of the equations [30, 31].

Under transformation (11), properties of the heat equation and corresponding properties of the Black-Scholes equation can be mapped back and forth. It turns out that nonclassical symmetries are among the properties that are mapped back and forth between the heat and Black-Scholes equations.

\section{Nonclassical Symmetries of the Heat Equation}

Consider the heat equation

$$
\Delta=u_{t}-u_{x x}=0
$$

and the associated invariance surface condition

$$
\psi=\tau u_{t}+\xi u_{x}-\eta=0
$$

The invariance condition (8) is used to derive governing equations for nonclassical symmetries of the heat equation. If we adopt the usual notation $u_{i}=\partial u / \partial x^{i}, u_{i_{1} i_{2}}=\partial^{2} u$ / $\partial x^{i_{1}} \partial x^{i_{2}}, i, i_{j}=1,2, \quad\left(x^{1}, x^{2}\right)=(x, t)$, and $\left(\xi^{1}, \xi^{2}\right)=(\xi, \tau)$, and let $D_{i}$ denote the total differential operator with respect to $x^{i}$ :

$$
D_{i}=\frac{\partial}{\partial x^{i}}+u_{i} \frac{\partial}{\partial u}+u_{i j} \frac{\partial}{\partial u_{j}}+u_{i j k} \frac{\partial}{\partial u_{j k}}+\cdots
$$

Then, $X^{(2)}$ is given by

$$
X^{(2)}=X+\eta_{i}^{(1)} \partial_{u_{i}}+\eta_{i_{1} i_{2}}^{(2)} \partial_{u_{i_{1} i_{2}}}, \quad i_{1}, i_{2}=1,2,
$$

where

$$
\eta_{i}^{(1)}=D_{i} \eta-\left(D_{i} \xi^{j}\right) u_{j}, \eta_{i_{1} i_{2}}^{(2)}=D_{i_{2}} \eta_{i_{1}}^{(1)}-\left(D_{i_{2}} \xi^{j}\right) u_{i_{1} j}, \quad i, i_{k}, j=1,2 .
$$

The use of a symbolic manipulation program to execute (8) and perform other algebraic manipulations is imperative in view of the involved nature of the attendant calculations. In this connection, we used MATHEMATICA [32]. The following system of governing equations for nonclassical symmetries of the heat equation was obtained:

$$
\begin{gathered}
\xi \tau^{2} \tau_{u u}-\xi_{u u} \tau^{3}=0, \\
3 \xi_{u u} \tau^{2} \eta-2 \xi_{u} \xi^{2} \tau+2 \xi_{x u} \xi \tau^{2}-2 \xi \tau \tau_{u u} \eta \\
-2 \xi^{2} \tau \tau_{x u}-\xi \tau^{2} \eta_{u u}=0,
\end{gathered}
$$

$$
\begin{gathered}
\xi^{2} \tau \tau_{u}=0, \\
\xi_{t} \xi^{2} \tau-4 \xi_{x u} \xi \tau-3 \xi_{u u} \tau \eta^{2}-\xi_{x x} \xi^{2} \tau+2 \xi_{u} \xi^{2} \eta \\
+2 \xi_{x} \xi^{3}+2 \xi^{2} \tau_{x u} \eta+\xi \tau_{u u} \eta^{2}+\xi^{3} \tau_{x x}-\xi^{3} \tau_{t} \\
+2 \xi^{2} \tau \eta_{x u}+2 \xi \tau \eta \eta_{u u}=0, \\
\xi^{2} \tau_{u} \eta+\xi^{3} \tau_{x}=0, \\
\xi_{x x} \xi^{2} \eta-\xi_{t} \xi^{2} \eta+2 \xi_{x u} \xi \eta^{2}+\xi_{u u} \eta^{3}+\xi^{3} \eta_{t}-\xi^{3} \eta_{x x} \\
-2 \xi^{2} \eta \eta_{x u}-\xi \eta^{2} \eta_{u u}=0 .
\end{gathered}
$$

A solution of this system leads to a nontrivial nonclassical symmetry of equation (13) if the invariant surface condition (14) is a necessary condition in the invariance condition (8). Furthermore, the obtained infinitesimals must lead to a reduction of the heat equation (13) to an ordinary differential equation (ODE) via the usual reduction method.

To simplify the determining equations, we typically make assumptions on the nature of the infinitesimals $\xi, \tau$, and $\eta$. Mansfield [33], one of the first to consider nonclassical symmetries of the heat equation, simplified the determining equations by considering two cases, when $\tau \neq 0$ (in which we can set $\tau=1$, without loss of generality) and when $\tau=0$. The thrust of this paper, however, is not about determination of nonclassical symmetries of the heat equation. Rather, it is about obtaining nonclassical symmetries of the Black-Scholes equation from nonclassical symmetries of the heat equation. So, we simply consider a number of arbitrary cases to obtain four nonclassical symmetries of the heat equation.

Case 1. $\xi=w(x), \tau=1$, and $\eta=0$.

In this case, all the governing equations (18)-(23) are satisfied except (21), which is reduced to the second-order ODE:

$$
w^{2}\left(2 w w^{\prime}-w^{\prime \prime}\right)=0 .
$$

The general solution of (24) is

$$
w=\frac{k_{1}\left(k_{2}-e^{2 k_{1} x}\right)}{k_{2}+e^{2 k_{1} x}},
$$

where $k_{1}$ and $k_{2}$ are arbitrary constants. We therefore have that a nonclassical symmetry arising from this case is

$$
X_{1}=\frac{k_{1}\left(k_{2}-e^{2 k_{1} x}\right)}{k_{2}+e^{2 k_{1} x}} \partial_{x}+\partial_{t},
$$

provided $k_{1} k_{2} \neq 0$.

Case 2. $\xi=0, \tau=w(x)$, and $\eta=1$.

For this nature of infinitesimals, the governing equations (18)-(23) are satisfied for an arbitrary function $w$. However, the heat equation (13) is reduced to an ODE via the reduction method only if $w$ solves the ODE 


$$
w^{\prime \prime}-\frac{2 w^{\prime 2}}{w}=0 .
$$

The general solution of which is

$$
w=\frac{k_{1}}{x+k_{2}},
$$

where $k_{1}$ and $k_{2}$ are arbitrary constants. Therefore, a nonclassical symmetry arising from this case is

$$
X_{2}=\frac{k_{1}}{x+k_{2}} \partial_{t}+\partial_{u},
$$

provided $k_{1} \neq 0$.

Case 3. $\xi=1, \tau=0$, and $\eta=w(x)$.

In this case, the governing equations (18)-(23) are satisfied provided the function $w$ satisfies $w^{\prime \prime}=0$, which leads to the nonclassical symmetry:

$$
X_{3}=\partial_{x}+\left(k_{1}+k_{2} x\right) \partial_{u},
$$

where $k_{1}$ and $k_{2}$ are arbitrary constants, with $k_{1}^{2}+k_{2}^{2} \neq 0$.

Case 4. $\xi=\sum_{0}^{1} a_{i} x^{i}, \tau=\sum_{0}^{1} b_{i} t^{i}$, and $\eta=\sum_{0}^{1} c_{i} u^{i}, a_{i}, b_{i}, c_{i}$ arbitrary.

In this case, all the governing equations (18)-(23) are satisfied except (21), which is reduced to the algebraic equation

$$
\left(2 a_{1}-b_{1}\right)\left(a_{0}+a_{1} x\right)^{3}=0 .
$$

Different classical and nonclassical symmetries of the heat equation may be derived from this case depending on choices of the parameters. If we set $a_{1}=b_{1 / 2}$, for example, we obtain

$$
X_{4}=\left(a_{0}+\frac{1}{2} b_{1}\right) \partial_{x}+\left(b_{0}+b_{1} y\right) \partial_{t}+\left(c_{0}+c_{1} u\right) \partial_{u},
$$

which is a nontrivial nonclassical symmetry of the heat equation provided $b_{1}^{2}+c_{0}^{2} \neq 0$.

\section{Derivation of Nonclassical Symmetries of the Black-Scholes Equation}

For simplicity, we suitably fix parameters in the nonclassical symmetries (26), (29), (30) and (32) and revert back to the original variables $z, y$, and $w$ of the heat equation (9) to obtain

$$
\begin{aligned}
& X_{1}=\left(\frac{1-e^{2 z}}{1+e^{2 z}}\right) \partial_{z}+\partial_{y}, \\
& X_{2}=\left(\frac{1}{z}\right) \partial_{y}+\partial_{w}, \\
& X_{3}=\partial_{z}+z \partial_{w}
\end{aligned}
$$

$$
X_{4}=2 \partial_{z}+\partial_{y}+\partial_{w}
$$

Each of these nonclassical symmetries of the heat equation is now mapped to a corresponding nonclassical symmetry of the Black-Scholes equation by the transformation

$$
\begin{aligned}
& x=\exp \left\{\frac{\sigma^{2}(z-y)}{2 \kappa}\right\}, \\
& t=-\frac{\sigma^{2} y}{2 \kappa^{2}}
\end{aligned}
$$

$$
u=w \exp \left\{-\frac{r \sigma^{2} y}{2 \kappa^{2}}\right\}
$$

which is the inversion of (11) with (w.l.o.g.) $\delta_{1}=\delta_{2}=0$ and $\delta_{3}=1$.

Under (37), any (classical or nonclassical) symmetry,

$$
X=\xi(z, y, w) \partial_{z}+\tau(z, y, w) \partial_{y}+\eta(z, y, w) \partial_{w},
$$

of the heat equation (9) is transformed into a corresponding symmetry of the Black-Scholes equation (1):

$$
\bar{X}=\bar{\xi}(x, t, u) \partial_{x}+\bar{\tau}(x, t, u) \partial_{t}+\bar{\eta}(x, t, u) \partial_{u},
$$

where

$$
\begin{aligned}
& \bar{\xi}(x, t, u)=\left.X(x)\right|_{(11)}, \\
& \bar{\tau}(x, t, u)=\left.X(t)\right|_{(11)}, \\
& \bar{\eta}(x, t, u)=\left.X(u)\right|_{(11)},
\end{aligned}
$$

with $\delta_{1}=\delta_{2}=0$ and $\delta_{3}=1$. We take the liberty to normalise the resulting vector fields when necessary in light of the property that $X$ and $\widetilde{X}$ are equivalent nonclassical symmetries if $\widetilde{X}=\Lambda(x, t, u) X$, provided $\Lambda(x, t, u)$ is a nonvanishing smooth function of its arguments (see [14], for example).

We obtain the following nonclassical symmetries of the Black-Scholes equation:

$$
\begin{aligned}
& Z_{1}=2 \kappa x^{4 \kappa / \sigma^{2}+1} \partial_{x}+\left(e^{4 \kappa^{2} t / \sigma^{2}}+x^{4 \kappa / \sigma^{2}}\right) \partial_{t}+r u\left(e^{4 \kappa^{2} t / \sigma^{2}}+x^{4 \kappa / \sigma^{2}}\right) \partial_{u}, \\
& Z_{2}=\kappa x \partial_{x}+\partial_{t}+\left(r u-\frac{4 \kappa^{3} e^{r t}(\ln x-\kappa t)}{\sigma^{4}}\right) \partial_{u} \\
& Z_{3}=\frac{\sigma^{2}}{2 \kappa} x \partial_{x}+\frac{2 \kappa e^{r t}(\ln x-\kappa t)}{\sigma^{2}} \partial_{u} \\
& Z_{4}=\kappa x \partial_{x}-\partial_{t}+\left(\frac{2 \kappa^{2} e^{r t}}{\sigma^{2}}-r u\right) \partial_{u}
\end{aligned}
$$

from the heat equation symmetries (33)-(36), respectively. 


\section{Invariant Solutions of the Black-Scholes Equation Obtained from the Nonclassical Symmetries}

Each of the nonclassical symmetries admitted by the Black-Scholes equation may be used to construct solutions of the equation. These will be new solutions of the Black-Scholes equation in the sense that they are not obtainable directly as invariant solutions associated with classical Lie point symmetries of the Black-Scholes equation. The construction of such solutions parallels the usual reduction method for classical Lie point symmetries. For each nonclassical symmetry $X$ of equation (2), one determines from solutions of the associated characteristic system,

$$
\frac{\mathrm{d} x}{\xi}=\frac{\mathrm{d} t}{\tau}=\frac{\mathrm{d} u}{\eta}
$$

two independent invariants $r=r(x, t, u)$ and $v=v(x, t, u)$ (with $v_{u} \neq 0$ ) of the associated group. The form of the invariant solution of equation (2) arising from $X$ is now obtained from $v=\varphi(r)$ for some unknown function $\varphi$, or

$$
u=\Theta(x, t),
$$

if we solve for $u$. Upon substitution of (46) into (2), we obtain an ODE that defines $\Theta$. The solution of this ODE completes the process.

Invariant solutions of the Black-Scholes equation obtained from the constructed nonclassical symmetries of the equation are presented below.

Solution 1. $\xi=2 \kappa \quad x^{4 \kappa / \sigma^{2}+1}, \tau=e^{4 \kappa^{2} t / \sigma^{2}}+x^{4 \kappa / \sigma^{2}}$, and $\eta=r u$ $\left(e^{4 \kappa^{2} t / \sigma^{2}}+x^{4 \kappa / \sigma^{2}}\right)$.

The solution of equation (1) arising from the nonclassical symmetry (41) is

$$
u(x, t)=e^{r t} w(\zeta), \zeta=\frac{1}{\kappa} \ln x-\frac{\sigma^{2}}{2 \kappa^{2}} \ln \left(e^{4 \kappa^{2} t / \sigma^{2}}-x^{4 \kappa / \sigma^{2}}\right)+2 t
$$

where $w$ satisfies the ODE

$$
2 \kappa^{2} w^{\prime}+\sigma^{2} w^{\prime \prime}=0 .
$$

The general solution of (48) is

$$
w=k_{1}+k_{2} \exp \left(-\frac{2 \kappa^{2} \zeta}{\sigma^{2}}\right)
$$

where $k_{1}$ and $k_{2}$ are arbitrary constants so that (47) and (49) constitute the invariant solution of the Black-Scholes equation associated with the nonclassical symmetry (41).

Solution 2. $\xi=\kappa x, \tau=1$, and $\eta=r u-4 \kappa^{3} e^{r t}(\ln x-\kappa t) / \sigma^{4}$.

The solution of equation (1) arising from the nonclassical symmetry (42) is

$$
u(x, t)=\frac{4 \kappa^{3} t e^{r t}(\kappa t-\ln x)}{\sigma^{4}}+e^{r t} w(\zeta), \zeta=x e^{-\kappa t},
$$

where $w$ satisfies the ODE

$$
\zeta^{2} w^{\prime \prime}+\zeta w^{\prime}-\frac{\left(2 r-\sigma^{2}\right)^{3}}{\sigma^{6}} \ln \zeta=0 .
$$

The general solution of (51) is

$$
w=k_{1}+k_{2} \ln \zeta-\frac{\left(\sigma^{2}-2 r\right)^{3}}{6 \sigma^{6}} \ln ^{3} \zeta
$$

where $k_{1}$ and $k_{2}$ are arbitrary constants so that (50) and (52) constitute the invariant solution of the Black-Scholes equation associated with the nonclassical symmetry (42).

Solution 3. $\xi=\sigma^{2} x /(2 \kappa), \tau=0$, and $\eta=2 \kappa e^{r t}(\ln x-\kappa t) / \sigma^{2}$.

The solution of equation (1) arising from the nonclassical symmetry (43) is

$$
u(x, t)=w(t)-\frac{2 \kappa^{2} e^{r t}(2 \kappa t-\ln x) \ln x}{\sigma^{4}},
$$

where $w$ satisfies the first-order ODE

$$
w^{\prime}-r w+e^{r t}\left[\left(\frac{2 \kappa^{3}}{\sigma^{2}}-\frac{4 \kappa^{3} r}{\sigma^{4}}\right) t+\frac{2 \kappa^{2}}{\sigma^{2}}\right]=0 .
$$

The general solution of (54) is

$$
w=e^{r t}\left(k_{1}+\frac{\kappa^{3}\left(2 r-\sigma^{2}\right) t^{2}}{\sigma^{4}}-\frac{2 \kappa^{2} t}{\sigma^{2}}\right),
$$

where $k_{1}$ is an arbitrary constant so that (53) and (55) constitute the invariant solution of the Black-Scholes equation associated with the nonclassical symmetry (43).

Solution 4. $\xi=\kappa x, \tau=-1$, and $\eta=2 \kappa^{2} e^{r t} / \sigma^{2}-r u$.

The solution of equation (1) arising from the nonclassical symmetry (44) is

$$
u(x, t)=e^{r t}\left(w(\zeta)-\frac{2 \kappa^{2} t}{\sigma^{2}}\right), \zeta=x e^{\kappa t},
$$

where $w$ satisfies the ODE

$$
\zeta^{2} w^{\prime \prime}+\frac{2(\kappa+r)}{\sigma^{2}} \zeta w^{\prime}-\frac{4 \kappa^{2}}{\sigma^{4}}=0 .
$$

The general solution of (57) is

$$
w=k_{1}+k_{2} \zeta^{2\left(\sigma^{2}-2 r\right) / \sigma^{2}}-\frac{\left(\sigma^{2}-2 r\right)}{2 \sigma^{2}} \ln \zeta,
$$

where $k_{1}$ and $k_{2}$ are arbitrary constants so that (56) and (58) constitute the invariant solution of the Black-Scholes equation associated with the nonclassical symmetry (44).

\section{Concluding Remarks}

In this paper, we sought to illustrate how equivalent transformations may be used to find nonclassical symmetries of differential equations. We have constructed new bonafide nonclassical symmetries of the Black-Scholes equation by using the well-established equivalence between this equation and the heat equation. A particular invertible 
point transformation between the Black-Scholes equation and the heat equation was exploited.

Nonclassical symmetries of the heat equation were firstly derived in the usual way, starting with determining equations generated from the invariance condition for nonclassical symmetries and then solving the equations in specific instances by making assumptions on the nature of the infinitesimals.

Four nonclassical symmetries of the heat equation were determined and transformed into four nontrivial nonclassical symmetries of the Black-Scholes equation. Clearly, additional ones may be constructed in a similar fashion. For completeness the constructed nonclassical symmetries were used to find solutions of the Black-Scholes equation via the reduction method. The solutions of the Black-Scholes equation communicated in this article are new in that they have not been reported before and cannot be constructed directly as invariant solutions from classical Lie point symmetries of the Black-Scholes equation.

\section{Data Availability}

The data used to support the findings of this study are available from the author upon request.

\section{Conflicts of Interest}

The author declares that there are no conflicts of Interest regarding the publication of this paper.

\section{Acknowledgments}

The author thanks the Directorate of Research Development and Innovation of Walter Sisulu University for continued financial support.

\section{References}

[1] M. C. Nucci, "Nonclassical symmetries as special solutions of heir-equations," Journal of Mathematical Analysis and Applications, vol. 279, no. 1, pp. 168-179, 2003.

[2] M. C. Nucci, "Iterating the nonclassical symmeteries method," Physica D: Nonlinear Phenomena, vol. 78, no. 1-2, pp. 124-134, 1994.

[3] F. Allassia and M. C. Nucci, "Symmetries and heir equations for the laminar boundary layer model," Journal of Mathematical Analysis and Applications, vol. 201, no. 3, pp. 911-942, 1996.

[4] M. S. Hashemi and M. C. Nucci, "Nonclassical symmetries for a class of reaction-diffusion equations: the method of heirequations," Journal of Nonlinear Mathematical Physics, vol. 20 , no. 1 , pp. $44-60,2013$.

[5] M. S. Hashemi, "On Black-Scholes equation; method of heirequations, nonlinear self-adjointness and conservation laws," Bulletin of the Iranian Mathematical Society, vol. 42, pp. 903-921, 2016.

[6] C. Temuer, L. Tong, and G. Bluman, "Some connections between classical and nonclassical symmetries of a partial differential equation and their applications," Mathematics, vol. 8, no. 4, p. 524, 2020.
[7] N. Bîlă and J. Niesen, "On a new procedure for finding nonclassical symmetries," Journal of Symbolic Computation, vol. 38, pp. 1523-1533, 2004.

[8] M. S. Bruzón and M. L. Gandarias, "Applying a new algorithm to derive nonclassical symmetries," Communications in Nonlinear Science and Numerical Simulation, vol. 13, no. 3, pp. 517-523, 2008.

[9] F. Black and M. Scholes, "The pricing of options and corporate liabilities," Journal of Political Economy, vol. 81, no. 3, pp. 637-654, 1973.

[10] G. Bluman and J. Cole, "The general similarity solution of the heat equation," Indiana University Mathematics Journal, vol. 18, no. 11, pp. 1025-1042, 1969.

[11] G. Cai, Y. Wang, and F. Zhang, "Nonclassical symmetries and group invariant solutions of Burgers-Fisher equations," World Journal of Modelling and Simulation, vol. 3, pp. 305-309, 2007.

[12] Z. Zhang and Y. Chen, "Classical and nonclassical symmetries analysis for initial value problems," Physics Letters A, vol. 374, no. 9, pp. 1117-1120, 2010.

[13] R. Naz, M. D. Khan, and I. Naeem, "Nonclassical symmetry analysis of boundary layer equations," Journal of Applied Mathematics, vol. 2012, Article ID 938604, 1 page, 2012.

[14] P. J. Olver and E. M. Vorob'ev, "Nonclassical and conditional symmetries," in CRC Handbook of Lie Group Analysis of Differential Equations, N. H. Ibragimov, Ed., vol. 3, pp. 291328, CRC Press, Boca Raton, FL, USA, 1996.

[15] G. W. Bluman and V. Shtelen, "Developments in similarity methods related to pioneering work of Julian Cole," in Mathematics Is for Solving Problems; Cook, V. Roytburd and M. Tulin, Eds., pp. 105-118, SIAM, Philadelphia, PA, USA, 1996.

[16] P. A. Clarkson, D. K. Ludlow, and T. J. Priestley, "The classical, direct, and nonclassical methods for symmetry reductions of nonlinear partial differential equations," Methods and Applications of Analysis, vol. 4, no. 2, pp. 173-195, 1997.

[17] G. W. Bluman and S. Kumei, Symmetries and Differential Equations, Springer-Verlag, Berlin, Germany, 1989.

[18] G. W. Bluman, A. F. Cheviakov, and S. C. Anco, Applications of Symmetry Methods to Partial Differential Equations, Springer, Berlin, Germany, 2010.

[19] L. V. Ovsiannikov, Group Analysis of Differential Equations, Academic Press, Cambridge, MA, USA, 1982.

[20] B. J. Cantwell, Introduction to Symmetry Analysis, Cambridge University Press, Cambridge, UK, 2002.

[21] P. E. Hydon, Symmetry Methods for Differential Equations: A Beginner's Guide, Cambridge University Press, Cambridge, UK, 2000.

[22] P. J. Olver, Applications of Lie Groups to Differential Equations, Springer-Verlag, Berlin, Germany, 1993.

[23] G. Baumann, Symmetry Analysis of Differential Equations with Mathematica, Springer, Berlin, Germany, 2000.

[24] R. K. Gazizov and N. H. Ibragimov, "Lie symmetry analysis of differential equations in finance," Nonlinear Dynamics, vol. 17, no. 4, pp. 387-407, 1998.

[25] G. W. Bluman, "On mapping linear partial differential equations to constant coefficient equations," SIAM Journal on Applied Mathematics, vol. 43, no. 6, pp. 1259-1273, 1983.

[26] G. W. Bluman and S. Kumei, "Symmetry-based algorithms to relate partial differential equations: I. Local symmetries," European Journal of Applied Mathematics, vol. 1, no. 3, pp. 189-216, 1990.

[27] W. Sinkala, "Two ways to solve, using Lie group analysis, the fundamental valuation equation in the double-square-root 
model of the term structure," Communications in Nonlinear Science and Numerical Simulation, vol. 16, no. 1, pp. 56-62, 2011.

[28] S. Anco, G. Bluman, and T. Wolf, "Invertible mappings of nonlinear PDEs to linear PDEs through admitted conservation laws," Acta Applicandae Mathematicae, vol. 101, no. 1-3, pp. 21-38, 2008.

[29] S. Kumei and G. W. Bluman, "When nonlinear differential equations are equivalent to linear differential equations," SIAM Journal on Applied Mathematics, vol. 42, no. 5, pp. 1157-1173, 1982.

[30] F. M. Mahomed, "Complete invariant characterization of scalar linear $(1+1)$ parabolic equations," Journal of Nonlinear Mathematical Physics, vol. 15, no. sup1, pp. 112-123, 2008.

[31] T. Aziz, A. Fatima, and C. M. Khalique, "Integrability analysis of the partial differential equation describing the classical bond-pricing model of mathematical finance," Open Physics, vol. 16, no. 1, pp. 766-779, 2018.

[32] Wolfram Research, Inc. Mathematica. Version 9.0, Wolfram Research, Champaign, IL, USA, 2012.

[33] E. L. Mansfield, "The nonclassical group analysis of the Heat equation," Journal of Mathematical Analysis and Applications, vol. 231, no. 2, pp. 526-542, 1999. 